\title{
2D SELF-ORGANIZED GRADIENT PERCOLATION MODEL FOR NUMERICAL SIMULATION OF IMPREGNATION IN POROUS MEDIA
}

\author{
C. B. TRANG ${ }^{1}$, T. SAYET ${ }^{1}$, A. BATAKIS ${ }^{2}$, E. BLOND ${ }^{1}$, E. DE BILBAO ${ }^{3}$ \\ ${ }^{1}$ Univ. Orléans, LaMé (EA7494 \\ Univ. Orléans, Univ. Tours, INSA CVL), France \\ \{cong-bang.trang, eric.blond, thomas.sayet\}@univ-orleans.fr \\ ${ }^{2}$ Univ. Orléans, Insitut Denis Poisson \\ UMR CNRS, Univ. Orléans, Univ Tours \\ athanasios.batakis@univ-orleans.fr \\ ${ }^{3}$ Univ. Orléans, CEMHTI \\ UPR 3079 CNRS, France \\ emmanuel.debilbao@univ-orleans.fr
}

\begin{abstract}
Reactive impregnation concerns many science and engineering areas, such as corrosion in the steel-making industry and chemical engineering. Furthermore, reactive impregnation can become dangerous in some applications. Simulating non-reactive impregnation with classical methods is the first step before computing reactive impreg++nation. However, existing numerical methods present problems such as high computational cost and spurious oscillation. To avoid these computational difficulties, we propose the Self-organized Gradient Percolation model. It is a numerical model based on probabilistic approaches and, in particular, on percolation methods. This work aims to present a 2D model based on the 1D developed model. The first results are free from spurious oscillation and drastically reduced the computational cost compared with the classical methods.
\end{abstract}

Keywords: Impregnation, Richards' equation, Porous media, Percolation

\section{INTRODUCTION}

Numerical modeling of the non-reactive impregnation process in porous material requires a multi-physics model with several material properties [1, 2]. Yet, it often demands extensive computing facilities, thereby leading to time-consuming computation. It also requires a fine mesh leading to spurious oscillations at the first few time steps affecting accuracy [4]. As a first attempt to find a way less time-consuming but still making sure the numerical accuracy, Selforganized Gradient Percolation (SGP) method has been successfully proposed to reproduce the Capillary Pressure Profiles over time without solving Richards' equation [1].

This study aims to extend the existing 1D SGP model for the non-reactive and unsaturated impregnation for a higher dimension (2D/3D). At a starting point, our current strategy only focuses on the 2D case. Indeed, we obtain the local saturation by interpolating a "cluster" based on the index of the considered local square (i.e., the interpolation-index method will be introduced in this paper). Moreover, the spread over time of that cluster is based on Classical Gradient Percolation with assumptions. Visualization and comparison with finite element method (FEM) to validate the implementation of the method are given in this paper.

\section{SELF-ORGANIZED GRADIENT PERCOLATION (SGP) METHOD}

Gradient Percolation Method is a probabilistic method reproducing the liquid's spread at a microscopic scale $[5,7]$. Two typical types of percolation methods are bond percolation and site percolation [5]. From physics standpoints, the site percolation model is a better candidate 
to perform the non-reactive unsaturated impregnation process, whose local square represents a local pore space having one of two states (let say "occupied" and "empty") [7].

Mathematically, the site percolation model is defined as the following: Considering a site $\mathbf{z}$ of the lattice $\mathbb{Z}^{2}$. Let $U(\boldsymbol{z})$ be a uniform random variable over $[0,1], P=P(\mathbf{z})$ be a real-valued function having range in $[0,1]$.

- $\quad$ Site $\mathbf{z}$ is occupied by liquid if $P(\mathbf{z}) \geq U(\mathbf{z})$;

- Otherwise, site $\mathbf{z}$ is empty.

The condition can understand that the site $\boldsymbol{z}$ has a probability $P(\boldsymbol{z})$ to be occupied. In simulation, we define a function Clust connecting to site $\mathbf{z}$. We say that $\operatorname{Clust}(\mathbf{z})=1$ if the site $\mathbf{z}$ is occupied, otherwise $\operatorname{Clust}(\mathbf{z})=0$.

\subsection{Self-Organized Gradient Percolation for $1 \mathrm{D}$ case}

Self-Organized Gradient Percolation method (SGP) in light of the Gradient Percolation Method has been proposed to reproduce Capillary Pressure Profiles overtime of the impregnation phenomena [6]. For the capillary rising test, we choose the function $P(\mathbf{z})$ to be a function of a local site (nodes in the sense of numerical analysis). Physics implies that the state of a local site is given by the capillary pressure determined by the difference between the force of the wetting and non-wetting phase of phenomena [3]. However, it is visible that the numerical simulations of the SGP method hav problems of continuity (Figure 3). Moreover, it is natural to ask how to define boundary conditions for the model. That is why the conyolution operator has been proposed to ensure continuity and consider various boundary conditions. The local saturation of each local site is calculated by two steps: (1) for the whole model and (2) usin

$$
S(\mathrm{z}, t)=X(\mathrm{z}, \mathrm{t}) * \delta(\mathrm{z})
$$
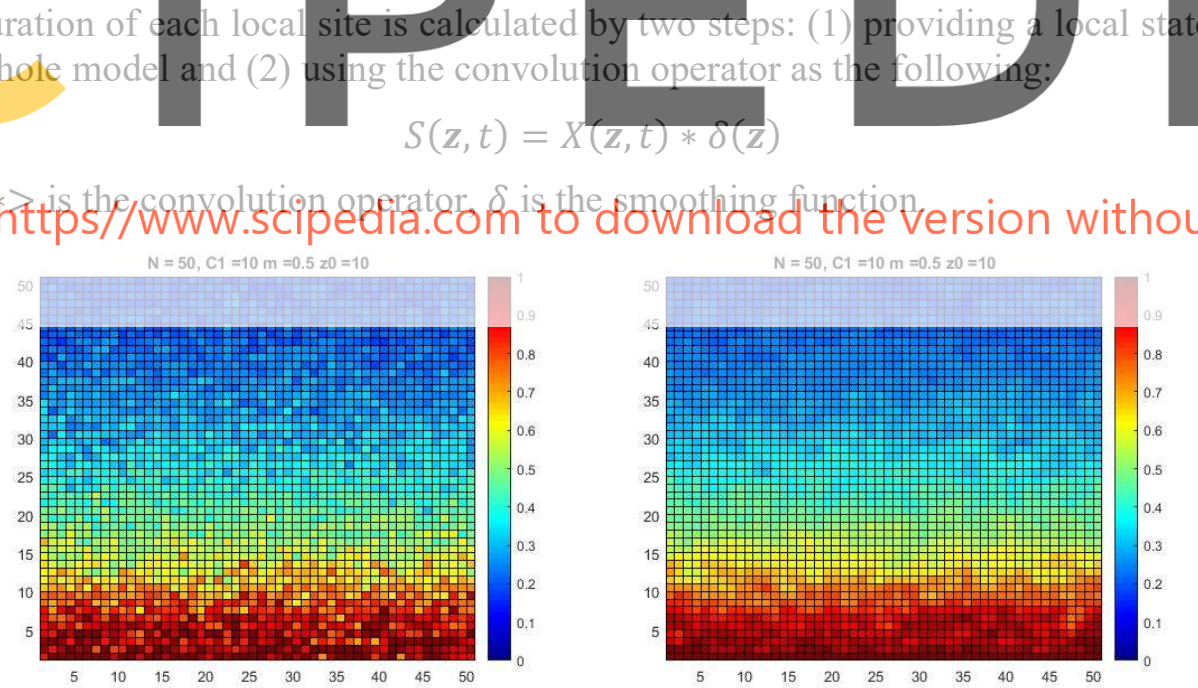

Figure 1: SGP model. Left: Before the convolution, there is a problem with continuity. Right: After the convolution. 


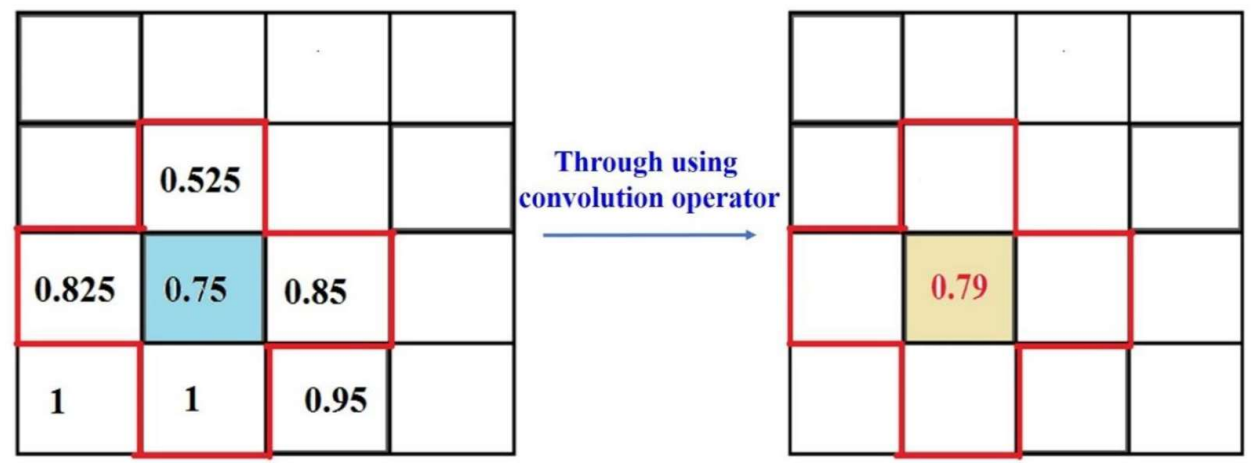

Figure 2: Convolution operator works in the local sites. Left: Before the convolution. Right: After the convolution.

From physics standpoints, on the first step time, the state function $X(z, t)$ is defined by [6]:

$$
X\left(P_{\text {cap }}(\mathbf{z}), t_{n}\right)=S_{r}+\left(S_{\text {max }}-S_{r}\right) e^{\left(-\frac{\left|P_{\text {cap }}(\mathbf{z})-P_{\text {cap }, t_{n}}^{S_{\text {max }}}\right|^{m}}{m \sigma_{n}^{m}}\right)}
$$

where $S_{r}$ and $S_{\max }$ are the residual and maximum saturation, respectively, $P_{c a p}$ is the local

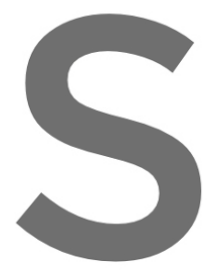

capillary pressure,

standard deviation

switching between

$m=2$ designate Laplace an

To reproduce the physical phenomenon, the

with time, following the phenomenon's physical laws. The relationship is proposed in tube-

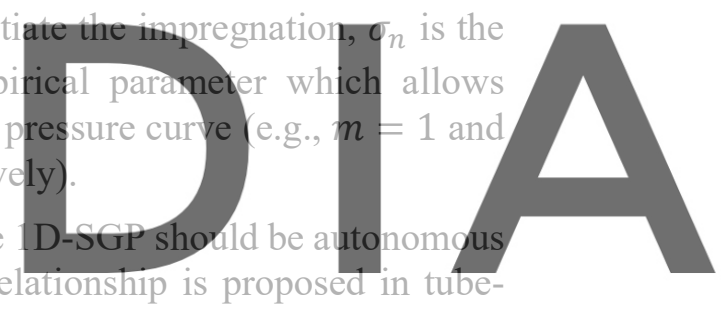

model [8] - non-gravity verșion:

$$
\begin{gathered}
v_{n}=A \frac{P_{\text {cap }, f i x}}{\sigma_{n}} \\
\sigma_{n+1}=\sigma_{n}+v_{n} d t_{n} \\
P_{c a p, n+1}^{S_{\max }}=P_{c a p, n}^{S_{\max }}+B v_{n} d t_{n}
\end{gathered}
$$

where $d t_{n}$ is the size of the step time, $A$ is a physical constant, $P_{\text {cap,fix }}$ is capillary pressure relating to tube model [8], and $B$ is the constant relating to the evolution of saturated zone.

SGP method has been validated [6] for 1D- impregnation case. In the next steps, the SGP method is therefore modified with extension to the $2 \mathrm{D}$ case.

\subsection{Self-Organized Gradient Percolation for 2D case}

Extending to the 2D SGP method is dealing with main challenges as follows:

- How to define the direction of the flow in the impregnation process?

- How to define boundary conditions according to the shape of the boundary?

This section aims to define the direction of the flow in the process.

With an initial condition Clust $_{1}$ on the domain such as the L-domain (Figure 5), at the time step $n+1$, our approach is to extend the SGP method as the following three steps: 


\section{a) First step:}

1. The probability value $P_{n+1}(\mathbf{z})$ at this time step is defined to be

$$
P_{n+1}(\mathbf{z})=\left(\text { Clust }_{n} * \delta_{1}\right)(\mathbf{z})
$$

where $C l u s_{n}$ is the cluster obtained by percolation method at time step $n, \delta_{1}$ is a smoothing function that will be chosen appropriately and $\langle *\rangle$ is a convolution operator. This assumption guarantees the practice of the simulation.

2. The cluster at the time step $n+1$ is calculated by a formula:

$$
\text { Clus }_{n+1}(\mathbf{z})=\left\{\begin{array}{lr}
1 & P_{n+1}(\mathbf{z}) \geq U_{n+1}(\mathbf{z}) \\
0 & \text { otherwise }
\end{array}\right.
$$

where $U_{n+1}(\mathbf{z})$ is a uniformly random variable depending on the site at time step $n+1$.

3. The cluster value Clust $_{n+1}(\boldsymbol{z})$ of site, $\boldsymbol{z}$ becomes one if there is a neighborhood $\mathbf{z}_{1}$ of that the site $\mathbf{z}$ (node) such that: $\operatorname{Clust}_{n}\left(\mathbf{z}_{1}\right)=1$. The liquid only flows from a site to its neighbor site.

4. If $\operatorname{Clust}_{n}(\mathbf{z})=1, \operatorname{Clust}_{n+1}(\mathbf{z})=1$. On microscopic scale, if the boundary is unchanged over time, there will be liquid from the boundary flowing to this site.

\section{To understand the method, consider a simple example:}
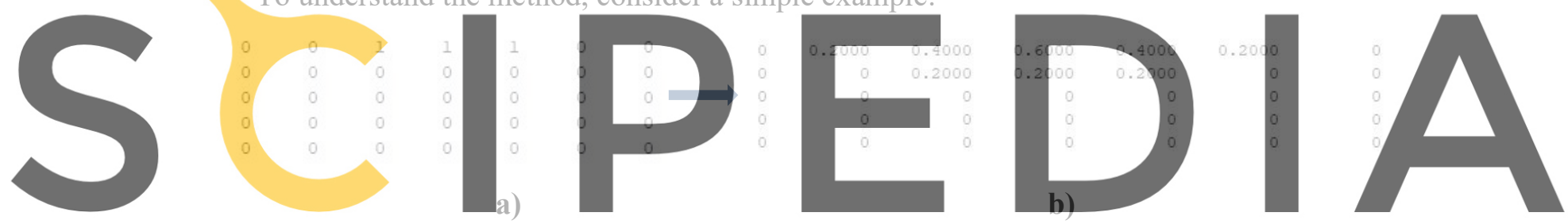

Register for free at https//www.scipedia.com to download the version without the watermark

c)

d)

Figure 3: Simple simulation to describe the method. a) Initial condition. b) Apply the convolution to a). c) The result of the method. d) Assumption 3. to c.

Let an initial condition Figure 3a, the sites having cluster value $\operatorname{Clust}_{1}(\mathbf{z})=1$ are the boundary condition. After that, we use the convolution to obtain Figure 3b. Classical Gradient Percolation is applied with probability in Figure 3b. For example, " 0.2 " means that the site has one over five chances to become one. The result of this process is Figure 3c. By assumption 3, we obtain Figure 3d.

Considering a finer mesh, we can achieve a better result being closer to reality. Mathematically, a cluster is a collection of sites (nodes) having Clust $_{n}=1$ in the sample of the above method at each time step $n$. In physics, this step is analog to the microscopic scale of impregnation. We will use the result of the first step to define the flow direction. 


\section{b) Second step:}

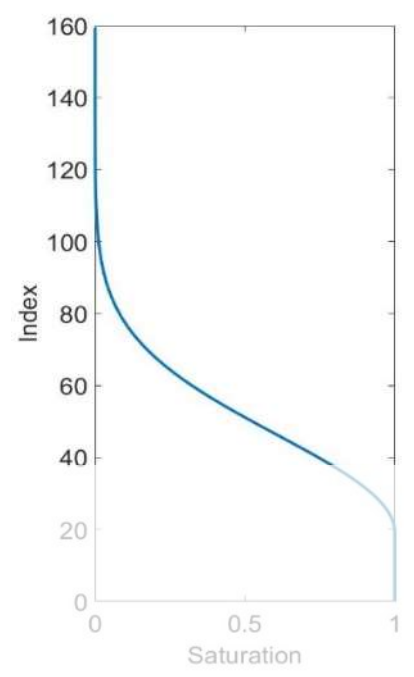

Figure 4: 1D SGP model, we define the index of saturation value to link node index. The uniform division is applied to the index.

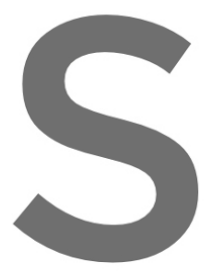

In practice, the macroscopic saturation value varies from 0 to function linking to Richards's equation to interpolate the cluster function from the 1D \$GP model (Khol 2018 step $n+1$ :

$1 D S G P_{n+1}($ index $):=1 D S G P(P$
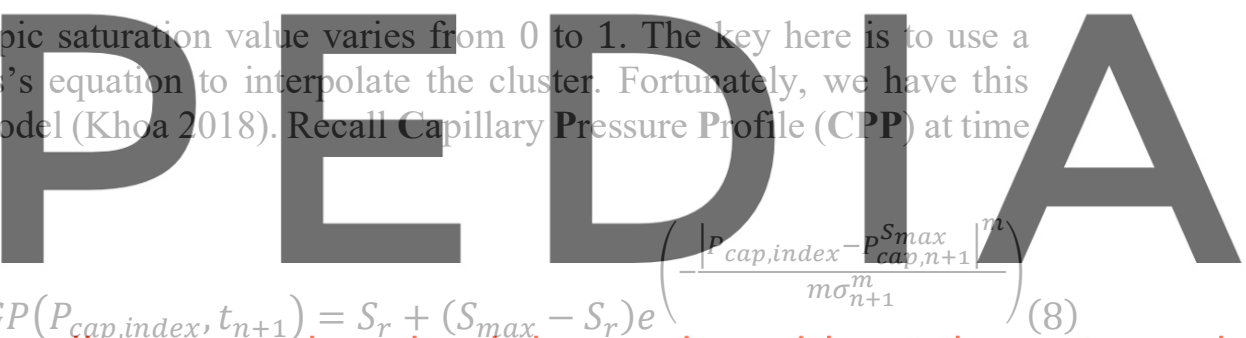
Register for free at https//www.scipedia.com to download the version without the watermark

c) Third step:
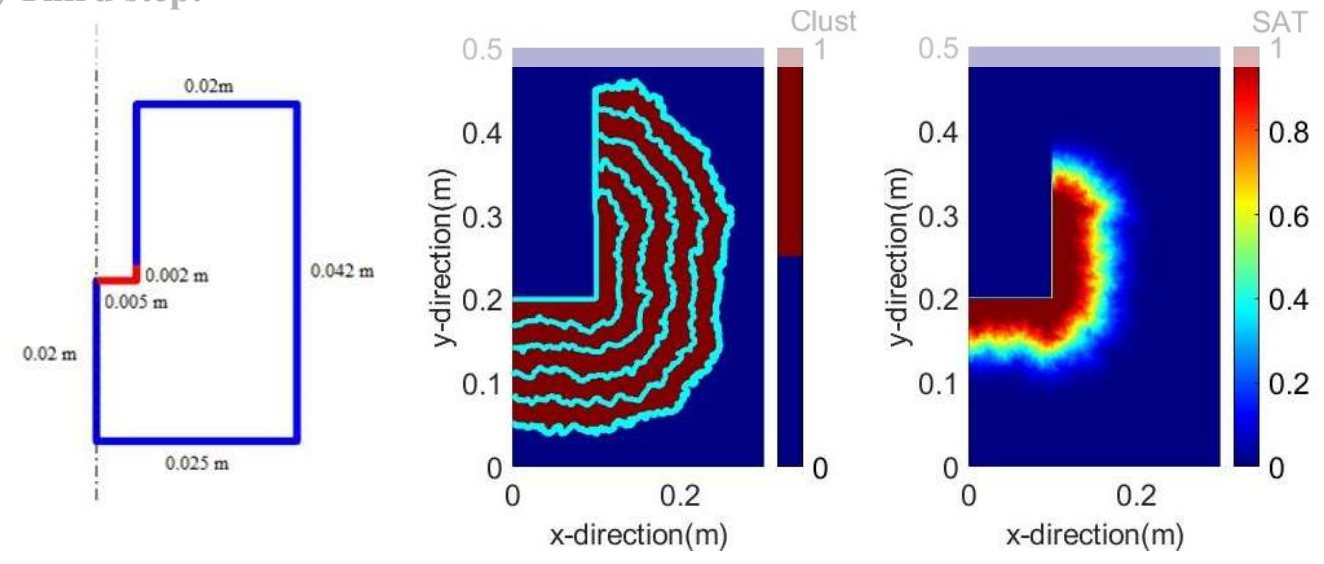

Figure 5: Left: Initial domain with boundary condition. Center: Result of Gradient Percolation method with the above assumptions in the first step (Cluster result). Right: Result when we apply interpolation-index method (Saturation result) 

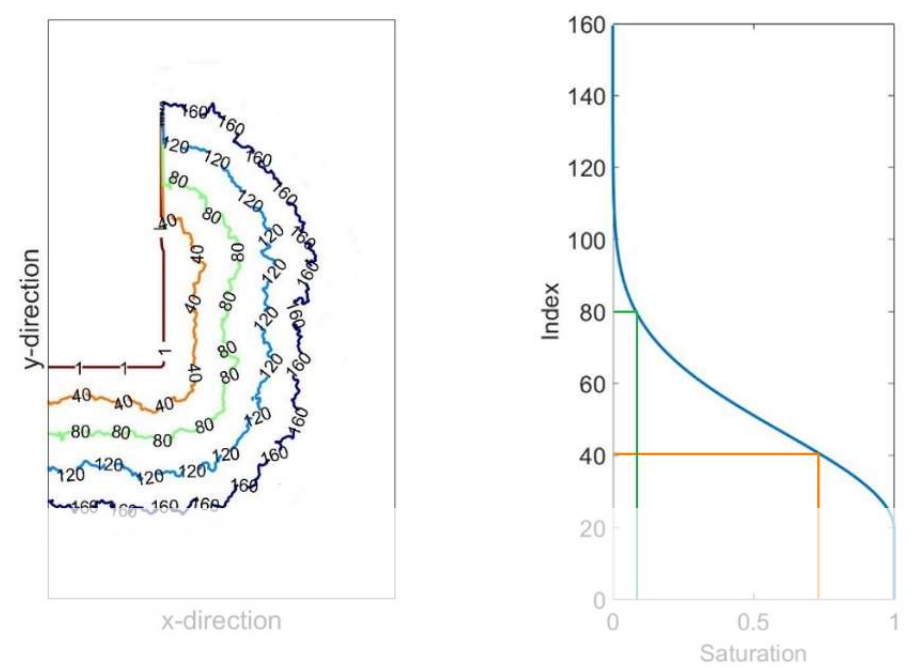

Figure 6: Left: The index of the cluster in time step $n+1$. Right: 1D SGP model. The "orange" isoline takes the value of "orange" saturation.

To achieve the macroscopic saturation, Interpolation-Index method is proposed as follows:

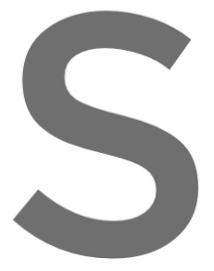

First, index function Clust $_{i}(\mathbf{z})=1$ and $C$ smallest value $k$ such 3 , the condition "Clust $t_{j}(\mathbf{z})$ As a result, the index
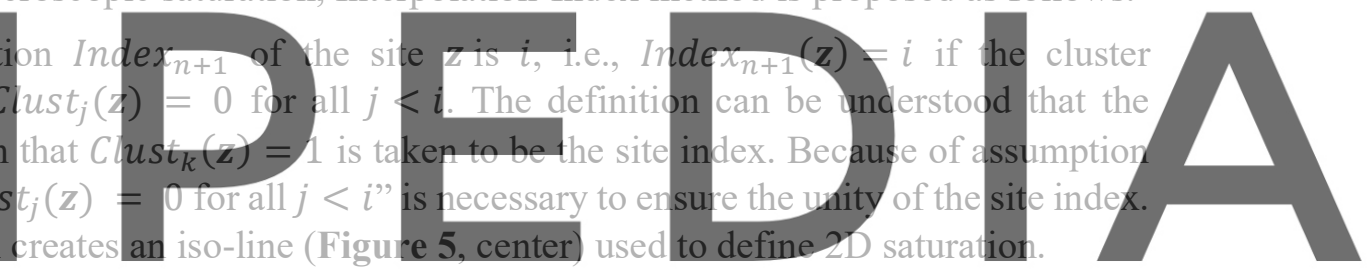

Second, we partition the Capillary Pressure Profile (Figure 4) with uniformly distributed

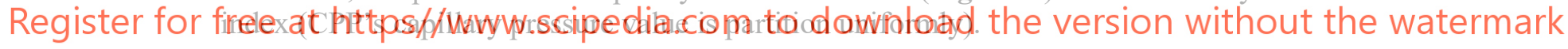

Finally, the $i$ th indexed sites take the value of the $i$ th element of the vector to obtain the saturation. (Figure 5 and Figure 6). Miore precisely, the 2D saturation $S_{n+1}$ at the site $\mathbf{z}$ has the value $1 D S G P_{n+1}(i)$, i.e. $S_{n+1}(\mathbf{z})=1 D S G P_{n+1}(i)$ if $\operatorname{Index}_{n+1}(\mathbf{z})=i$.

\subsection{Boundary conditions}

This paper only uses two types of boundary conditions: drained and undrained boundary conditions, which may or may not allow the liquid to impregnate the sample (mathematically, Neumann condition). In the first period $\left[t_{0}, t_{1}\right]$, depending on the problem, we choose a drained boundary condition (for example, two surfaces as Figure 5). 


\subsection{Algorithm}

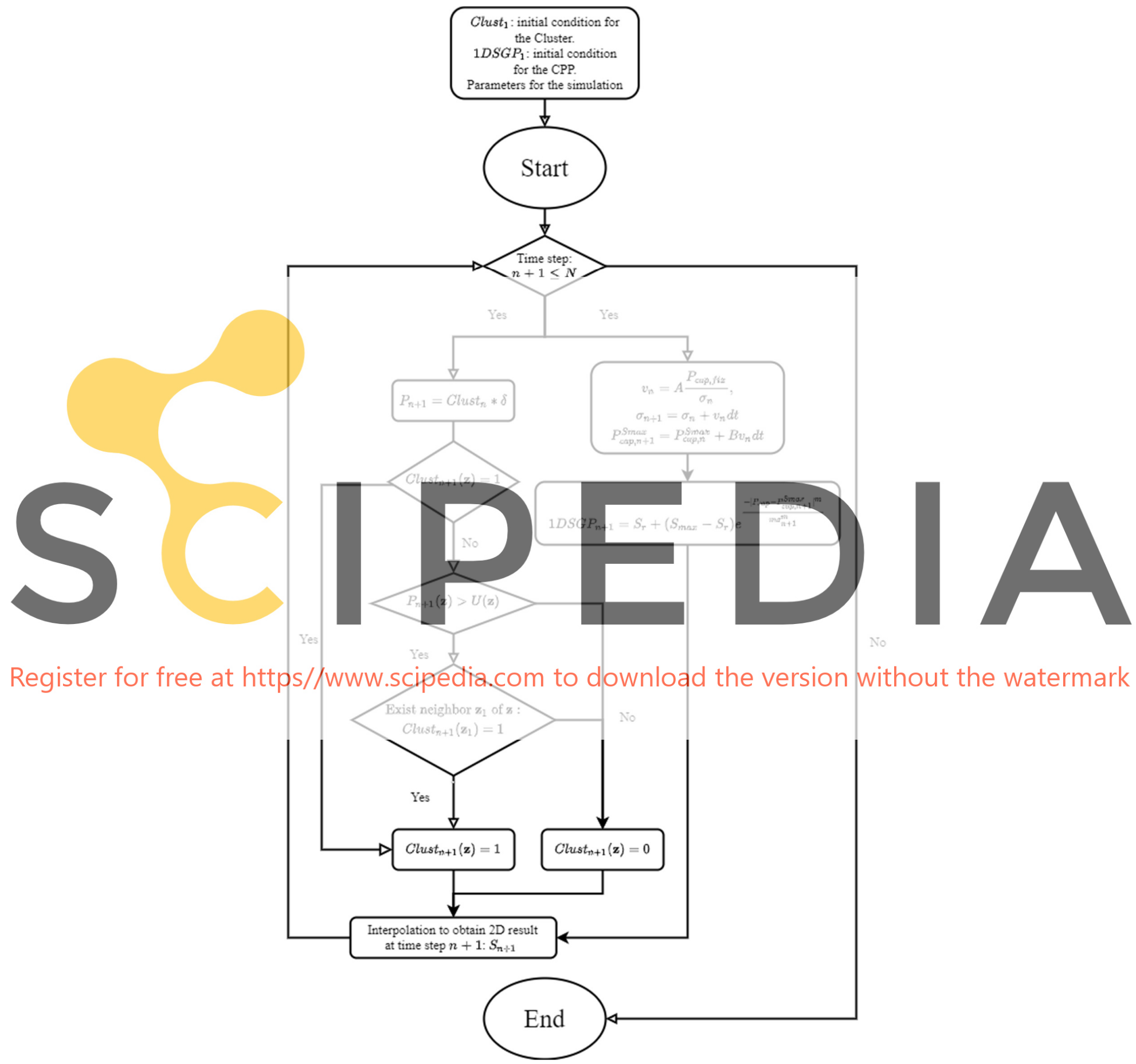

Figure 7: Algorithm of the interpolation- index method and SGP model

where $U$ is the uniformly random variable in the percolation method, and $N$ is the maximum step time for the simulation. The condition $n \leq N$ can be replaced with a better condition in the future result. At the moment, we still try to find a good way to stop the method. 


\section{APPLICATION}

\subsection{Domain description and material data}

In this section, we consider an axisymmetric domain with L-boundary. The blue boundaries are subjected to the Neumann condition, whose flux is chosen to be equal to zero (the liquid incapable of flowing from the domain to the outside in this region). The red one allows the liquid to impregnate. Furthermore, we define the saturation $\boldsymbol{S}$ always equals to one in this region. The sample's height and width of the sample, respectively, are $\mathbf{0 . 0 4 2} \mathbf{~ m}$ and $\mathbf{0 . 0 2 5} \mathbf{~ m}$, the horizontal impregnated surface (drained boundary condition) where the liquid flows through it is $0.002 \mathrm{~m}$, the vertical impregnated surface is $0.005 \mathrm{~m}$. We use uniform square-mesh of size $\mathbf{1 0}^{-\mathbf{5}} \mathbf{m}$ in the simulation.
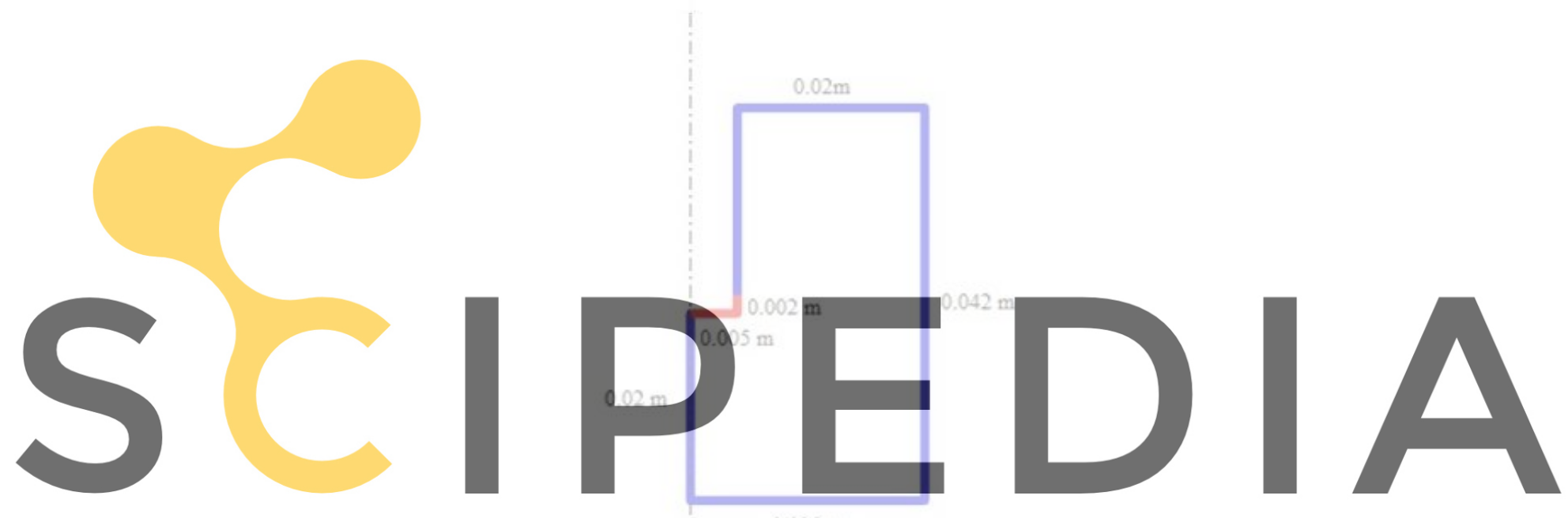

Register for free at https//www.scipedia.com to download the version without the watermark

Figure 8: Axisymmetric L-boundary domain $\boldsymbol{\Omega}$, liquid impregnates through two red zones.

Recall, Finite Element Method is solving Richards' equation [1,3] to simulate the impregnation. The equation is given by:

$$
\phi \frac{\partial S}{\partial t}=-\operatorname{div}\left(\frac{K_{\text {int }} \psi(S)}{\eta} \nabla\left(p_{\text {cap }}-\rho g z\right)\right)
$$

where $S$ is the saturation of the liquid (dimensionless), $\rho$ is the density of the liquid $\left(\mathrm{kg} / \mathrm{m}^{3}\right)$, $g$ is the gravitational constant $\left(\mathrm{m} / \mathrm{s}^{2}\right), \psi(S)$ is the relative permeability (dimensionless), which can be a cubic function or more complicated, $K_{i n t}$ is the intrinsic permeability $\left(\mathrm{m}^{2}\right), \eta$ is the viscosity of the liquid (Pa.s), $\phi$ is the porosity of the sample (dimensionless). In several cases, the capillary pressure is assumed to depend on saturation $p_{\text {cap }}=p_{\text {cap }}(S)$ which is determined by many authors $[1,3]$ (such as van-Genuchten, Brooks and Corey, etc.). In this simulation, the van-Genuchten model is used for determining this relation:

$$
p_{c a p}(S)=p_{0}\left(S^{\frac{1}{i}}-1\right)^{1-i}
$$


where $p_{0}$ is the reference pressure $(\mathrm{Pa})$ and $i$ is a parameter of the model (dimensionless).

For SGP simulation, the constant $A$ is related to the kinetic of the impregnation process. According to viscosity $\eta$ and intrinsic permeability $K_{\text {int }}$, we propose:

$$
A=\frac{K_{i n t}}{\eta} \text {. }
$$

Table 1: Parameters for the simulation

\begin{tabular}{|c|c|c|c|}
\hline Parameter & Value & Unit & Description \\
\hline$\phi$ & 0.2 & dimensionless & $\begin{array}{c}\text { Porosity. It links to the porous } \\
\text { volume of the sample }\end{array}$ \\
\hline$K_{\text {int }}$ & $9.5 * 10^{-14}$ & $m^{2}$ & $\begin{array}{c}\text { Intrinsic permeability. It } \\
\text { relates to the "speed" of the } \\
\text { impregnation process. }\end{array}$ \\
\hline$\gamma=\rho g$ & 12348 & $\frac{N}{m^{3}}$ & $\begin{array}{c}\text { Specific weight. It is for } \\
\text { characterizing the "mass" of } \\
\text { liquid. }\end{array}$ \\
\hline$p_{0}$ & 1.48 & $P a \cdot s$ & $\begin{array}{c}\text { Viscosity It relates to the } \\
\text { interior friction of the liquid } \\
\text { Reference pressure. It is for } \\
\text { controlling the capillary } \\
\text { pressure's magnitude in thd } \\
\text { van-Genuchten model. }\end{array}$ \\
\hline
\end{tabular}

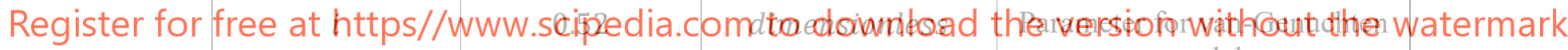

\begin{tabular}{|c|c|c|c|}
\hline$S_{r}$ & 0.1 & dimensionless & Minimum value of saturation \\
\hline$S_{\text {max }}$ & 1 & dimensionless & Maximum value of saturation \\
\hline$A=\frac{K_{\text {int }}}{\eta}$ & $6.4189 * 10^{-14}$ & $\frac{m^{2}}{P a \cdot s}$ & $\begin{array}{c}\text { SGP 1D model parameter.It } \\
\text { controls the "speed" of the } \\
\text { impregnation in SGP. The } \\
\text { mobility of liquid, i.e., the } \\
\text { ratio of the permeability and } \\
\text { the liquid viscosity. }\end{array}$ \\
\hline$p_{\text {cap_fix }}$ & 20000 & Pa & $\begin{array}{c}\text { SGP 1D model parameter. } \\
\text { This parameter relates to the } \\
\text { tube model [8]. }\end{array}$ \\
\hline$m$ & 1 & dimensionless & $\begin{array}{c}\text { SGP 1D model parameter. It } \\
\text { relates to the curve's shape in } \\
\text { the 1D model. }\end{array}$ \\
\hline
\end{tabular}




\subsection{Numerical result of 2D-SGP method}

This section is about a comparison between the saturation of the two methods.
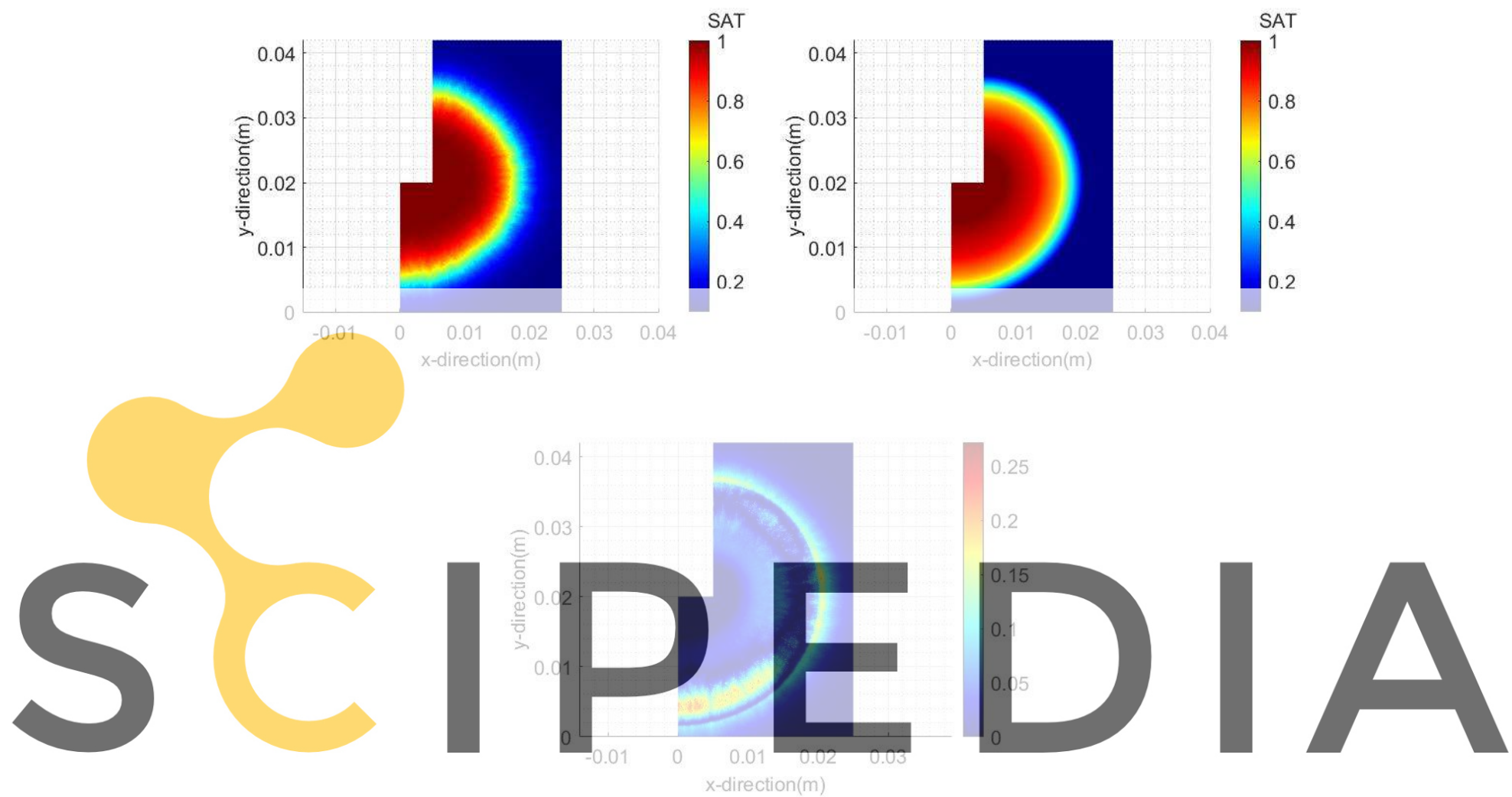

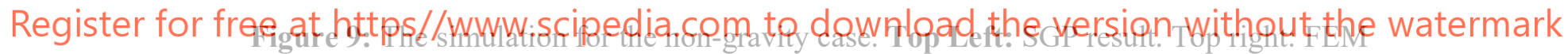
result. Bottom: Absolute error for the simulation between SGP and FEM on the local scale.

The local error formula: $\operatorname{Error}(z)=\operatorname{abs}(\operatorname{SGP}(z)-\operatorname{FEM}(z))$

The SGP method computation is obtained by running software on CPU: i5-8350U $1.7 \mathrm{GHz}$ with 16GB. We use the same input data for the simulation of the SGP method and FEM.

Table 2: CPU - time. Number of nodes: 94376

\begin{tabular}{|c|c|}
\hline Method & CPU time \\
\hline SGP & $392.8 \mathrm{~s}$ cluster $+4.5 \mathrm{~s}$ index \\
\hline FEM & $6916.4 \mathrm{~s}$ \\
\hline
\end{tabular}

There is two CPU time in 2D SGP model. The first is the CPU-time to calculate the cluster. The second is calculating the interpolation index method (including 1D SGP evolving. No spurious oscillations are affecting accuracy in the result and drastically reduce the CPU-time of the simulation. The reason is we do not solve Richards's equation at every time step. The 2D SGP model describes impregnation phenomena through the evolution of Gradient Percolation's result Clust and 1D SGP curve linking to the increment of $\sigma$ at every time step. 
The result shows the differential mechanics of the two methods. In the vertical boundary surface, a part of liquid impregnating the sample flows up (more precisely, moving vertically).

In FEM, the liquid's part flowing up has a speed slower than the one flowing vertically. When impregnating the sample, the liquid has to share some of its amounts in the vertical direction. The amount of this liquid is less than the one moving horizontally.

On the other hand, in the 2D SGP model, the liquid has approximately the same speed in every direction. With a site having the cluster value equals to one, its neighborhood has the same probability to becomes one. For a long time, the movement mostly the same in all directions. In the 2D SGP model, the cluster defines the direction of the liquid's flow.

In physics, the FEM simulation is more precise to reality [1,4]. Hence, to obtain the property "share amount of liquid" in FEM for the 2D SGP model, further work is needed.

\subsection{Average saturation curve}

The main result of this paper is in this section. The two methods, surprisingly, are the same in this method.

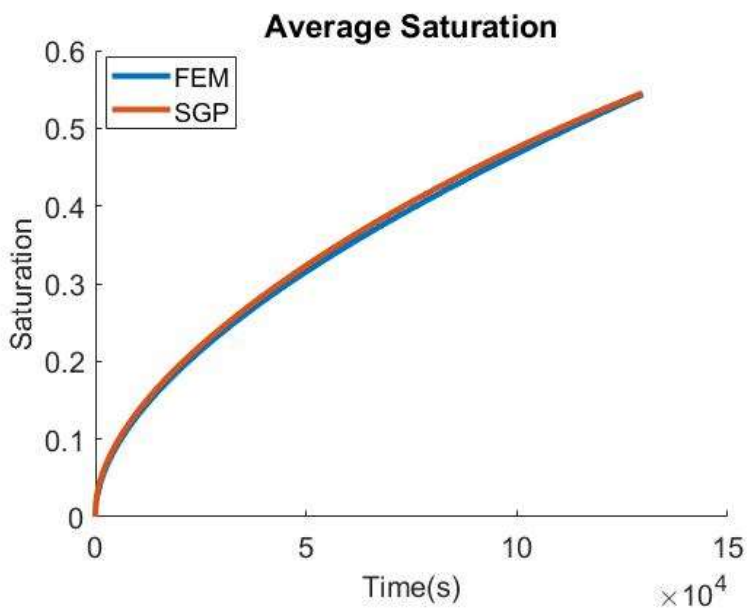

Figure 10: The average saturation of the SGP model and FEM.

The average saturation $\bar{S}$ is computed in domain $\Omega$ by the following formula:

$$
\bar{S}=\frac{1}{|\Omega|} \int_{\Omega} S d x
$$

where $|\Omega|$ is the volume of the domain $\Omega$.

These first results show a good agreement between SGP and FEM. We can explain this agreement is the liquid's compensation at the top of the sample and the bottom.

\section{CONCLUSION}

In this work, we study the 2D SGP method to simulate the impregnation process. The two main problems to extend to 2D are finding the 2D flow direction of liquid and adding the shape of boundary condition to the model. We can obtain the flow direction by using the Classical Gradient Percolation method and convolution operator. The initial results are promising in 
drastically reducing computational cost (CPU time). The accuracy of results is quite well for simulating the impregnation process (in average saturation curve).

However, when comparing with FEM in local nodes, the difference between the two methods is extensive. The problem comes from the sharing amount of liquid property. Also, the link of numerical time to real-time (time of phenomena) is still unknown. The future work will focus on fixing those problems and extend this method to gravity case and reactive impregnated case.

\section{REFERENCES}

[1] J. Bear and Y. Bachmat, Introduction to Modeling of Transport Phenomena in Porous Media. Dordrecht: Springer Netherlands, 1990.

[2] E. Blond, N. Schmitt, F. Hild, P. Blumenfeld, and J. Poirier, "Effect of Slag Impregnation on Thermal Degradations in Refractories," J American Ceramic Society, vol. 90, no. 1, pp. 154-162, Jan. 2007, doi: 10.1111/j.1551-2916.2006.01348.x.

[3] O. Coussy, "Mechanics and Physics of Porous Solids," p. 297.

[4] L. Pan, A. W. Warrick, and P. J. Wierenga, "Finite element methods for modeling water flow in variably saturated porous media: Numerical oscillation and mass-distributed schemes," Water Resources Research, vol. 32, no. 6, pp. 1883-1889, Jun. 1996, doi: 10.1029/96WR00753.

[5] G. Grimmett, Percolation. New York, NY: Springer New York, 1989.

[6] A. K. Nguyen, E. Blond, T. Sayet, A. Batakis, E. de Bilbao, and M. D. Duong, "Selforganized gradient percolation method for numerical simulation of impregnation in porous media," Computer Methods in Applied Mechanics and Engineering, vol. 344, pp. 711-733, Feb. 2019, doi: 10.1016/j.cma.2018.10.027.

[7] P. Nolin, "Critical exponents of planar gradient percolation," The Annals of Probability, vol. 36, no. 5, pp. 1748-1776, Sep. 2008, doi: 10.1214/07-AOP375.

[8] C. W. Extrand, "Forces, pressures and energies associated with liquid rising in nonuniform capillary tubes," Journal of Colloid and Interface Science, vol. 450, pp. 135-140, Jul. 2015, doi: $10.1016 /$ j.jcis.2015.03.007. 